\section{Perfect Reconstruction QMF Banks for Two-Dimensional Applications}

\author{
P. P. VAIDYANATHAN
}

\begin{abstract}
A theory is outlined whereby it is possible to design a $M \times N$ channel two-dimensional quadrature mirror filter hank which has perfect reconstruction property. Such a property ensures freedom from aliasing, amplitude distortion, and phase distortion. The method is based on a simple property of certain transfer matrices, namely the losslessness property.
\end{abstract}

\section{INTRODUCTION}

The application of subband coding techniques for images has recently received considerable attention [1]-[4]. Vetterli [1] has extended the idea of quadrature mirror filtering [5] for the two-dimensional case. The usefulness of such techniques has been well demonstrated in recent contributions by Woods and O'Neil [2].

The purpose of this correspondence is to introduce certain methods whereby two-dimensional quadrature mirror filter (QMF) banks can be designed with complete freedom from all linear distortions (namely, aliasing, amplitude, and phase distortions [8]). Our results here are extensions of the one-dimensional results in [7]; the presentation here can, however, be understood in a self-contained manner, as the developments do not depend on those in [7]. A two-dimensional QMF band with $M N$ channels [1]-[4] is basically a parallel interconnection of $M N$ branches of the form shown in Fig. 1. The branch labeled $(m, n)$ takes the input signal $x\left(n_{1}, n_{2}\right)$, forms a subband signal by passing through the two-dimensional filter $H_{m n}\left(z_{1}, z_{2}\right)$ (analysis filters), and decimates the filtered signal by the factor $(M, N)$, where $M$ and $N$ are the decimation ratios in the horizontal and vertical directions, respectively. At the synthesis end, the signal is interpolated, filtered by the synthesis filters $F_{m n}\left(z_{1}, z_{2}\right)$ (to remove the images [6]), and then recombined. The operations of the decimators are described as $r_{m n}\left(n_{1}, n_{2}\right)=v_{m n}\left(M n_{1}, N n_{2}\right)$. The interpolators are described by $t_{m n}\left(n_{1}, n_{2}\right)=r_{m n}\left(n_{1} / M, n_{2} / N\right)$ if $n_{1}$ and $n_{2}$ are multiples of $M$ and $N$, respectively, and $t_{m n}\left(n_{1}, n_{2}\right)=0$ otherwise. In the transform domain, the decimator and interpolator are described, respectively, by the input-output relations $R_{m n}\left(z_{1}, z_{2}\right)=(1 / M N) \sum_{k=0}^{M-1} \sum_{l=0}^{N-1} V_{m n}\left(z_{1}^{1 / M} W_{M}^{k}, z_{2}^{1 / N} W_{N}^{l}\right)$ (where $W_{M}=e^{-2 \pi j / M}$, etc.), and $T_{m n}\left(z_{1}, z_{2}\right)=R_{m n}\left(z_{1}^{M}, z_{2}^{N}\right)$. Accordingly, the reconstructed output signal $\hat{x}\left(n_{1}, n_{2}\right)$ (which is the sum of the $M N$ signals $p_{m n}\left(n_{1}, n_{2}\right)$ in Fig. 1$)$ is related to the input $x\left(n_{1}, n_{2}\right)$ by

$$
\begin{aligned}
\hat{X}\left(z_{1}, z_{2}\right)=\frac{1}{M N} \sum_{k=0}^{M-1} \sum_{l=0}^{N-1} X\left(z_{1} W_{M}^{k}, z_{2} W_{N}^{l}\right) & \\
& \cdot \sum_{m=0}^{M-1} \sum_{n=0}^{N-1} H_{m n}\left(z_{1} W_{M}^{k}, z_{2} W_{N}^{l}\right) F_{m n}\left(z_{1}, z_{2}\right) .
\end{aligned}
$$

The terms in (1), except those corresponding to $k=l=0$, represent the effect of aliasing. If aliasing is somehow canceled by appropriate choice of the synthesis filters $F_{m n}\left(z_{1}, z_{2}\right)$, we are left

Manuscript received February 26, 1987. This work was supported in part by the National Science Foundation Grant DCI 8552579, and in part by Caltech's programs in Advanced Tcchnology Grant sponsorcd by Aerojet General, General Motors, GTE, and TRW.

The author is with the Department of Electrical Engineering, California Institute of Technology, Pasadena, CA 91125.

IEEE Log Number 8714985.

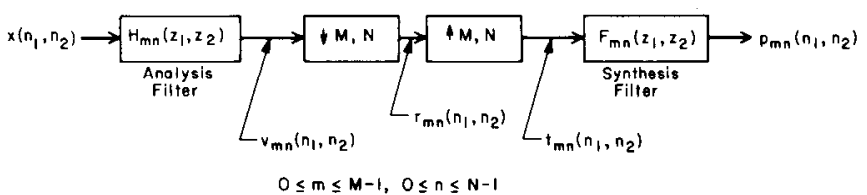

Fig. 1. The $(m, n)$ th branch of the $M \times N$ band 2-D QMF bank.

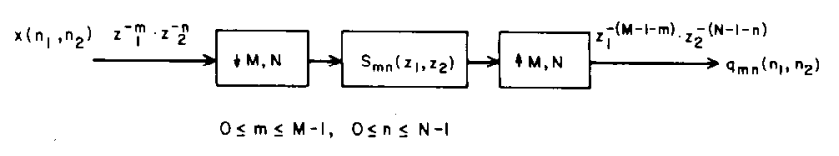

Fig. 2. The $(m, n)$ th branch of the "conceptual" structure.

with the distortion function

$$
\begin{aligned}
\hat{X}\left(z_{1}, z_{2}\right) / X\left(z_{1}, z_{2}\right) & =T\left(z_{1}, z_{2}\right) \\
= & \frac{1}{M N} \sum_{m=0}^{M-1} \sum_{n=0}^{N-1} H_{m n}\left(z_{1}, z_{2}\right) F_{m n}\left(z_{1}, z_{2}\right) .
\end{aligned}
$$

If $T\left(z_{1}, z_{2}\right)$ is a linear-phase (FIR) function, there is no phase distortion; if, on the other hand, it is an all-pass (IIR) function, there is no amplitude distortion. Finally, if $T\left(z_{1}, z_{2}\right)$ is a pure delay, i.e., $T\left(z_{1}, z_{2}\right)=c z_{1}^{-L_{1}} z_{2}^{-L_{2}}$, then we have perfect reconstruction, i.e.,

$$
\hat{x}\left(n_{1}, n_{2}\right)=c x\left(n_{1}-L_{1}, n_{2}-L_{2}\right) .
$$

In order to understand how aliasing can be completely canceled, and how perfect reconstruction can be eventually attained, let us first consider the conceptual structure of Fig. 2, which we shall eventually relate to Fig. 1. Assume that we have a parallel connection of $M N$ branches of the form shown in Fig. 2 (with $0 \leqslant m \leqslant M-1$ and $0 \leqslant n \leqslant N-1)$ and let $\hat{y}\left(n_{1}, n_{2}\right)$ denote the output (which is the sum of the $M N$ signals $q_{m n}\left(n_{1}, n_{2}\right)$ ). The quantity $\hat{Y}\left(z_{1}, z_{2}\right)$ is then equal to

$$
\begin{aligned}
\frac{z_{1}^{-(M-1)} z_{2}^{-(N-1)}}{M N} & \sum_{k=0}^{M-1} \sum_{l=0}^{N-1} X\left(z_{1} W_{M}^{k}, z_{2} W_{N}^{\prime}\right) \\
& \cdot \sum_{m=0}^{M-1} \sum_{n=0}^{N-1} S_{m n}\left(z_{1}^{M}, z_{2}^{N}\right) W_{M}^{-k m} W_{N}^{-l n} .
\end{aligned}
$$

The signal $\hat{y}\left(n_{1}, n_{2}\right)$ is thus free from aliasing if (and only if) all the terms in (4) are zero except those corresponding to $k=l=0$. Accordingly, the system is alias-free if and only if $S_{m n}\left(z_{1}, z_{2}\right)$ is independent of $m$ and $n$. Letting $S_{m n}\left(z_{1}, z_{2}\right)=S\left(z_{1}, z_{2}\right)$ for all $m, n$, we then have

$$
\hat{Y}\left(z_{1}, z_{2}\right) / X\left(z_{1}, z_{2}\right)=z_{1}^{-(M-1)} z_{2}^{-(N-1)} S\left(z_{1}^{M}, z_{2}^{N}\right) .
$$

This result enables us to construct various types of QMF banks as shown below.

\section{Perfect Reconstruction QMF BanKS}

If $S\left(z_{1}, z_{2}\right)$ in (5) is a pure delay, we have perfect reconstruction. Now consider Fig. 3(a), where we have inserted two $M N \times$ $M N$ transfer matrices $\boldsymbol{E}\left(z_{1}, z_{2}\right)$ and $\boldsymbol{R}\left(z_{1}, z_{2}\right)$ into our conceptual circuit of Fig. 2. (The figure is drawn for the case of $M=N=2$, for convenience.) If the matrices are such that $\boldsymbol{R}\left(z_{1}, z_{2}\right)=\boldsymbol{E}^{-1}\left(z_{1}, z_{2}\right)$, then we still satisfy the perfect reconstruction property (i.e., (5) holds with $S\left(z_{1}, z_{2}\right)$ ). If the transfer matrices are now moved past the decimators and interpolators (according to standard rules [6]), this results in the equivalent 


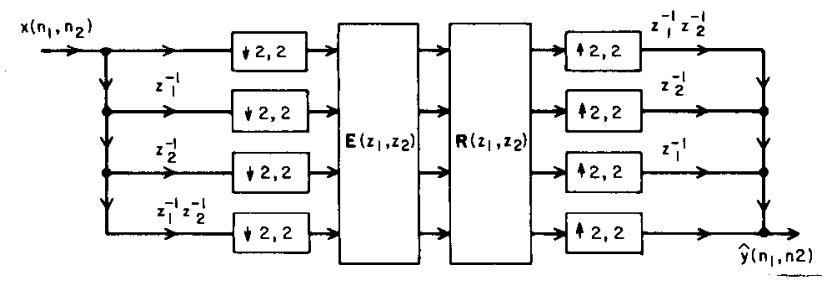

(a)

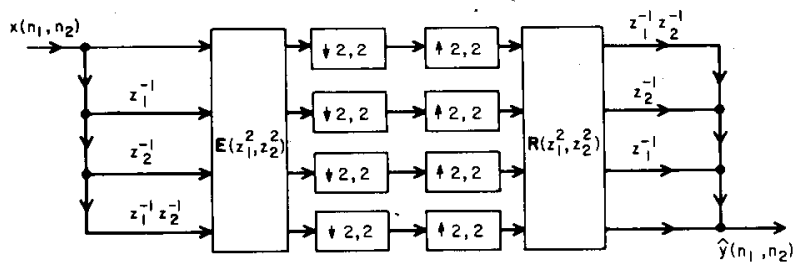

(b)

Fig. 3. (a) Inserting transfer matrices $\boldsymbol{E}\left(z_{1}, z_{2}\right)$ and $\boldsymbol{R}\left(z_{1}, z_{2}\right)$ into the conceptual structure. (b) Moving the decimators and interpolators to obtain an equivalent structure.

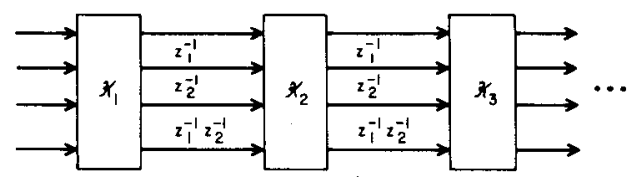

Fig. 4. A method for forcing $E\left(z_{1}, z_{2}\right)$ to be unitary. Here, the transfer matrix of the cascaded lattice structure is taken to be $E\left(z_{1}, z_{2}\right)$.

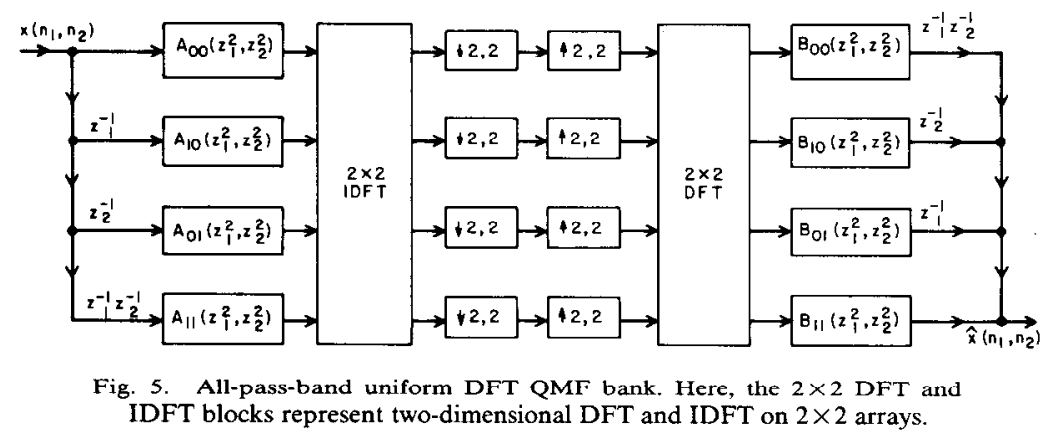

structure of Fig. 3(b). (Even though the rules in [6] are for the $1-D$ case, it is easily verified that similar results hold for the 2-D case.) By comparison with the QMF bank of Fig. 1, we see that Fig. 3(b) represents a standard QMF bank with

$$
\begin{aligned}
H_{r s}\left(z_{1}, z_{2}\right)= & \sum_{m=0}^{M-1} \sum_{n=0}^{N-1} E_{r+s M, m+n M}\left(z_{1}^{M}, z_{2}^{N}\right) z_{1}^{-m} z_{2}^{-n} \\
F_{r s}\left(z_{1}, z_{2}\right)= & \sum_{m=0}^{M-1} \sum_{n=0}^{N-1} R_{m+n M, r+s M}\left(z_{1}^{M}, z_{2}^{N}\right) \\
& \cdot z_{1}^{-(M-1-m)} z_{2}^{-(N-1-n)} .
\end{aligned}
$$

Thus, the transfer function $H_{r s}\left(z_{1}, z_{2}\right)$ has polyphase components [1]-[4] given by $E_{i j}\left(z_{1}, z_{2}\right)$, where $i=r+s M, j=m+n M$.

Given a set of analysis filters, we can therefore find the matrix $\boldsymbol{E}\left(z_{1}, z_{2}\right)$ and then invert it to obtain $\boldsymbol{R}\left(z_{1}, z_{2}\right)$ so that the synthesis filters for perfect reconstruction can be found. This approach, however, usually gives rise to synthesis filters of very high order which, in addition, are not guaranteed to be stable. A second approach is to restrict $H_{m n}\left(z_{1}, z_{2}\right)$ so that $\boldsymbol{E}\left(e^{j \omega_{1}}, e^{j \omega_{2}}\right)$ is unitary for all $\omega_{1}, \omega_{2} \cdot{ }^{1}$ Under this condition, if we choose $\boldsymbol{R}\left(z_{1}, z_{2}\right)$ to be equal to $\boldsymbol{E}^{T}\left(z_{1}^{-1}, z_{2}^{-1}\right)$, then we still have a perfect reconstruction system. If the analysis filters are FIR, then the entries of $\boldsymbol{E}\left(z_{1}, z_{2}\right)$ are FIR, and, hence, $\boldsymbol{R}\left(z_{1}, z_{2}\right)$ is FIR, with entries of the same length. However, $\boldsymbol{R}\left(z_{1}, z_{2}\right)$ is typically non-

\footnotetext{
${ }^{1}$ Such matrices $E\left(z_{1}, z_{2}\right)$ are said to be lossless, analogous to the one-dimen sional case [7].
}

causal; if this is undesirable, appropriate amounts of delays can be inserted without destroying the perfect reconstruction property.

In summary, if the analysis filters are FIR and such that the polyphase component matrix is unitary for $z_{1}=e^{j \omega_{1}}, z_{2}=e^{j \omega_{2}}$, then it is easy to find a set of FIR synthesis filters of the samc length (as the analysis filters), leading to perfect reconstruction. In order to force such unitariness, we have to structurally constrain $\boldsymbol{E}\left(z_{1}, z_{2}\right)$. For example, with $M=N=2$, Fig. 4 shows a way of doing this, where the matrices $\mathscr{K}$ are orthogonal. The transfer matrix of the cascaded lattice structure in Fig. 4 is itself taken to be $\boldsymbol{E}\left(z_{1}, z_{2}\right)$. If the coefficients of these matrices are optimized such that the analysis filters (given by (6)) have "good" stopbands, then a good perfect reconstruction system is automatically ensured.

\section{Comments on Separability}

If a perfect reconstruction system (which is of course free from aliasing) is designed in the above manner, $H_{m n}\left(z_{1}, z_{2}\right)$ does not have to be separable. This can be demonstrated as follows for the $M=N=2$ case, by taking the special case where $\boldsymbol{E}\left(z_{1}, z_{2}\right)$ is a constant matrix $E$ with the first row equal to $c, c, c, 2 c$, where $c=1 / \sqrt{7}$. The remaining rows of $\boldsymbol{E}$ can always be chosen such that $\boldsymbol{E}$ is orthogonal. With this choice, we have $H_{00}\left(z_{1}, z_{2}\right)=c(1$ $\left.+z_{1}^{-1}+z_{2}^{-1}+2 z_{1}^{-1} z_{2}^{-1}\right)$, which is nonseparable, and at the same time we have the perfect reconstruction property (in particular, aliasing has been eliminated). 


\section{AllPASS-BASED QMF BANKS}

One disadvantage with FIR. QMF banks is the requirement of high filter order for a given performance (such as stopband attenuation). It is possible to use IIR filters $H_{m n}\left(z_{1}, z_{2}\right)$, $F_{m n}\left(z_{1}, z_{2}\right)$ in such a way that aliasing is canceled, and such that $T\left(z_{1}, z_{2}\right)$ in (2) is allpass. This leaves behind some phase distortion, which can subsequently be compensated for by cascading with allpass equalizers. If such a scheme is practicable in a certain application, then the results of Section I offer a method for doing this. Fig. 5 demonstrates such a scheme for $M=N=2$, where $A_{m n}\left(z_{1}, z_{2}\right)$ and $B_{m n}\left(z_{1}, z_{2}\right)$ are all-pass functions such that $S\left(z_{1}, z_{2}\right)=A_{m n}\left(z_{1}, z_{2}\right) B_{m n}\left(z_{1}, z_{2}\right)$ is independent of $m, n$. As a result, the structure is equivalent to a parallel combination of the branches in Fig. 2 with $S_{m n}\left(z_{1}, z_{2}\right)$ independent of $m, n$. Aliasing is therefore absent, and $T\left(z_{1}, z_{2}\right)$ given by the RHS of (5) is allpass. Notice that the analysis and synthesis banks correspond to the uniform-DFT type. For example, with $M=N=2$, we have $H_{10}\left(z_{1}, z_{2}\right)=H_{00}\left(-z_{1}, z_{2}\right), H_{01}\left(z_{1}, z_{2}\right)=$ $\mathrm{H}_{00}\left(z_{1},-z_{2}\right), H_{11}\left(z_{1}, z_{2}\right)=H_{00}=\left(-z_{1},-z_{2}\right)$.

\section{CONCLUSIONS}

The design of transfer functions $H_{m n}\left(z_{1}, z_{2}\right)$ such that $\boldsymbol{E}\left(z_{1}, z_{2}\right)$ remains unitary should be done by computer-aided optimization by formulating an objective function which reflects the filtering quality of $H_{m n}\left(z_{1}, z_{2}\right)$. Since the objective function to be minimized is a nonlinear function, and since the responses are twodimensional, the design time may ultimately limit the sharpness and stopband attenuation of the analysis filters. For the design of the all-pass-based structure in Fig. 5, a similar computer-aided technique is required. But the IIR scheme has fewer parameters to optimize as compared to the perfect reconstruction FIR structure. Some of these design problems are currently under study.

\section{ACKNOWLEDGMENT}

The author is thankful to a reviewer for making certain important and useful remarks about the separability issue. Also, interesting discussions with V. C. Liu, graduate student at Caltech, are gratefully acknowledged.

\section{REFERENCES}

[1] M. Vetterli, "Multi-dimensional sub-band coding: Some theory and algorithms," Signal Processing, vol. 6, pp. 97-112, Apr. 1984.

[2] J. W. Woods and S. D. O'Neil, "Subband coding of images," IEEE Trans. Acoust., Speech, Signal Process., vol. 34, pp. 1278-1288, Oct. 1986.

[3] A. V. Brandt, "Subband coding of videoconference signals using quadrature mirror filtérs," in Proc. IASTED Conf. on Applied Signal Processing and Digital Filtering (Paris), June 1985.

[4] G. Wackersreuther, "On two-dimensional polyphase filter banks," IEFE Trans. Acoust., Speech, Signal Process., vol. 34, pp. 192-199, Feb. 1986.

[5] A. Croisier, D. Esteban, and C. Galand, "Perfect channel splitting by use of interpolation/decimation/tree decomposition techniques," presented at Int. Conf. on Info. Sci. and Sys., Patras, Greece, 1976.

[6] R. E. Crochiere and L. R. Rabiner, Multirate Digital Signal Processing. Englewood Cliffs, NJ: Prentice-Hall, 1983.

[7] P. P. Vaidyanathan, "Theory and design of $M$-channel maximally decimated quadrature mirror filters with arbitrary $M$ having perfectreconstruction property," Tech. Rep., California Institute of Technology, Apr. 1986 (also to be published in IEEE Trans. ASSP, Apr. 1987).

[8] M. J. T. Smith and T. P. Barnwell III, "A unifying framework for analysis/synthesis systems based on maximally decimated filter banks," in Proc. IEEE Int. Conf. on Acoust., Speech, Signal Process. (Tampa, FL), Mar. 1985, pp. 521-524.

\section{Split Vector Radix 2-D Fast Fourier Transform}

\author{
SOO-CHANG PEI AND JA-LIN WU
}

Abstract - The split vector radix is used to develop a 2-D fast Fourier transform (FFT) algorithm; it is performed "in-place," and requires no matrix transpose operation. This method greatly improves the conventional vector radix 2-D FFT; an overall saving of about 23 percent in complex multiplications for a typical $2048 \times 2048$ array could be obtained.

\section{INTRODUCTION}

The two-dimensional (2-D) discrete Fourier transform (DFT) is an important tool in digital image processing. Traditionally, the "row-column" method has been used to compute the 2-D fast Fourier transform (FFT) by taking 1-D FFT row-wise and column-wise; however, the time-consuming matrix transpose is needed in this row-column transform. Rivard, Harris, and McClellan [1], [2] use the vector radix to perform the decimation in both rows and columns simultaneously. The 2-D DFT is decomposed successively into several smaller 2-D DFT's until, ultimately, only trivial 2-D DFT's need to be evaluated. This vector radix method saves 25 percent in complex multiplications, and also avoids the matrix transpose operation.

The split radix has been proposed for 1-D FFT computations by Duhamel and Hollman [3], [4]. This algorithm has the advantage of being performed "in-place" in an FFT-like structure, and requires the lowest number of multiplications and additions for length $N=2^{n}$. Recently, Sorensen, Heideman, and Burrus have developed an efficient Fortran program on computing the split radix FFT [5]. Also, this algorithm can be used to efficiently calculate the fast Hartley transform [6], [7].

In this paper, we use the split vector decomposition to develop a 2-D fast Fourier transform; it greatly improves the conventional vector radix 2-D FFT. An overall saving of about 23 percent in complex multiplications for a typical $2048 \times 2048$ array could be obtained.

\section{Split Vector Radix AlgorithM}

The two-dimensional discrete Fourier transform is defined as

$$
X\left(k_{2}, k_{2}\right)=\sum_{n_{1}=0}^{N-1} \sum_{n_{2}=0}^{N-1} x\left(n_{1}, n_{2}\right) W_{N}^{n_{1} k_{1}} W_{N}^{n_{2} k_{2}}
$$

where

$$
W_{N}=\exp (-j 2 \pi / N)
$$

In the conventional vector radix 2-D FFT, we decompose the indices $-k_{1}, k_{2}$ simultaneously into four groups (see Fig. 1):

$$
\begin{array}{ll}
X\left(2 k_{1}, 2 k_{2}\right) & \text { even-even } \\
X\left(2 k_{1}, 2 k_{2}+1\right) & \text { even-odd } \\
X\left(2 k_{1}+1,2 k_{2}\right) & \text { odd-even } \\
X\left(2 k_{1}+1,2 k_{2}+1\right) & \text { odd-odd. }
\end{array}
$$

Manuscript received March 9, 1987.

S.-C. Pei is with the Department of Electrical Engincering, National Taiwan University, Taipei, Taiwan, R.O.C.

J.-L. Wu is with the Department of Electrical Engineering, Tatung Institute of Technology, Taipei, Taiwan, R.O.C.

IEEE Log Number 8714983 . 